\title{
Factores explicativos de la rentabilidad de las microempresas: Un estudio aplicado al sector comercio
}

\section{Factors explaining the profitability of microenterprises: A study applied to the commerce sector}

Fernando José Zambrano-Farías

Universidad Internacional del Ecuador, Guayaquil, Ecuador

Universidad de Guayaquil, Guayaquil, Ecuador

fezambranofa@uide.edu.ec

iD https://orcid.org/0000-0001-6384-3353

Carlos Iván Rivera-Naranjo

Escuela Superior Politécnica del Litoral, Guayaquil, Ecuador

cirivera@espol.edu.ec

iD https://orcid.org/0000-0003-4194-1387

Dennise Ivonne Quimi-Franco

Universidad de Guayaquil, Guayaquil, Ecuador

dennise.quimif@ug.edu.ec

iD https://orcid.org/0000-0002-5527-6245

Emilio Javier Flores-Villacrés

Universidad de Guayaquil, Guayaquil, Ecuador

emilio.floresvi@ug.edu.ec

iD https://orcid.org/0000-0003-1402-1462

Recepción: 01/10/2021 | Aceptación: 12/11/2021 | Publicación: 25/11/2021

Cómo citar (APA, séptima edición):

Zambrano-Farías, F.J., Rivera-Naranjo, C.I., Quimi-Franco, D.I., y Flores-Villacís, E.J. (2021).

Factores explicativos de la rentabilidad de las microempresas: Un estudio aplicado al sector comercio. INNOVA Research Journal, 6(3.2), 63-78. https://doi.org/10.33890/innova.v6.n3.2.2021.1974

\section{Resumen}

El presente estudio tiene como objetivo determinar los factores que explican la rentabilidad económica de las microempresas que pertenecen al sector comercio de la provincia del Guayas 
mediante un modelo de regresión lineal múltiple con datos suministrados por 788 empresas que reportaron sus estados financieros a la Superintendencia de Compañías, Valores y Seguros en el año 2019. Los resultados de estudio mostraron que el endeudamiento, el nivel de liquidez y el tamaño tienen una relación negativa y significativa con la rentabilidad. Este estudio también muestra que la rotación de activos, el capital de trabajo y el impacto de los gastos administrativos tienen una incidencia directa con el rendimiento sobre activo. Esta investigación pone de manifiesto la importancia de la información contable y financiera para explicar el comportamiento de la rentabilidad económica. Las conclusiones de este estudio pretenden ofrecer orientaciones a las empresas sobre las estrategias que deben adoptar para mejorar su rendimiento.

Palabras claves: rentabilidad; liquidez corriente; endeudamiento; microempresas; Guayas.

\begin{abstract}
The present study aims to determine the factors that explain the economic profitability of microenterprises belonging to the commerce sector in the province of Guayas through a multiple linear regression model with data provided by 788 companies that reported their financial statements to the Superintendence of Companies, Securities and Insurance in 2019. The study results showed that indebtedness, liquidity level and size have a negative and significante relationship with profitability. This study also shows that asset turnover, working capital and the impact of administrative costs have a direct impact on return on assets. This research highlights the importance of accounting and financial information in explaining the behaviour of economic profitability. The conclusions of this study aim to provide guidance to companies on the strategies they should adopt to improve their performance.
\end{abstract}

Keywords: profitability; current liquidity; indebtedness; microenterprises; Guayas.

\title{
Introducción
}

El principal objetivo de las empresas es obtener beneficios. Una empresa puede determinar si se consiguen beneficios cuando se evalúan sus resultados en función de la rentabilidad y se comparan estos resultados con los previstos al inicio del ejercicio. La teoría afirma que una empresa que tiene una elevada rentabilidad tiene más probabilidades de crecer, mientras que una empresa que genera poca rentabilidad supondrá una disminución del valor de sus acciones (Ali et al., 2019).

Con el objetivo de incrementar la rentabilidad, una empresa debe considerar una planificación estratégica que le permita administrar de manera eficiente sus operaciones y su flujo de caja. Sin embargo, el maximizar sus beneficios y alcanzar la rentabilidad deseada puede verse afectado por muchos factores, los mismos que son de carácter interno o asociados al entorno en el que se desenvuelven.

La identificación de los factores que afectan la variación de la rentabilidad de las empresas es un tema que ha provocado un auge de investigaciones en la última década. Entre los factores que se ha identificado en la literatura están la liquidez, el nivel de endeudamiento, el tamaño de la empresa (Evans, 1987; Garg et al., 2018; Handoko et al., 2019; Mursalini et al., 2017). En la actualidad, la importancia de la liquidez, el endeudamiento y el tamaño de la empresa y su incidencia en la rentabilidad ha generado un profundo debate. Una revisión de la literatura muestra 
que los resultados de las investigaciones previas no coinciden sobre cómo estos factores se relacionan con la rentabilidad de las empresas.

Garg et al. (2018) afirma que la administración de la liquidez y la rentabilidad es de gran importancia en el escenario actual, principalmente, para la decisión de la gestión financiera. Por su parte, Rasiah et al. (2014) señala que el tamaño de la empresa incide en la variación de rentabilidad y concluye que no existe una compensación identificable entre el crecimiento y rentabilidad.

Gran parte de las investigaciones (Franco et al., 2020; Jamil et al., 2015; Janda et al., 2011; Vivel-Búa et al., 2016; Zambrano Farías et al., 2021)están enfocadas a estudiar la rentabilidad de la empresas en su conjunto y son escasos los estudios acerca del rendimiento de las compañías categorizados por tamaño, normalmente se han realizado trabajos en contexto de las pymes o mipymes. Por tanto, es necesario que se realicen investigaciones que permitan conocer el comportamiento del desempeño empresarial de las microempresas por ser el conjunto más numeroso e incidente en el crecimiento económico de una sociedad.

A pesar de que, en Ecuador las microempresas conforman el $90.89 \%$ de la estructura empresarial y su contribución a la generación de empleo es importante, existe una cantidad limitada de estudios que tratan sobre la rentabilidad corporativa. En base a lo expuesto, el presente estudio pretende identificar los factores que inciden en la rentabilidad económica de las microempresas que pertenecen al sector de comercio al por mayor y menor de la provincia del Guayas. Los resultados de este estudio proporcionan directrices a las empresas sobre la mejor medida a utilizar para evaluar su rendimiento.

La investigación está estructura de la siguiente forma. Se inicia una sucinta revisión literaria sobre estudios relacionados a la rentabilidad de las empresas; luego se explica la selección de la muestra que es una base de datos conformada por las empresas cuya información se ajusta a los objetivos de esta investigación, además de una descripción de las variables que se utilizarán en el estudio. En el siguiente apartado se presentan los resultados obtenidos por el modelo propuesto y finalmente se presentan las conclusiones derivadas de esta investigación.

\section{Marco teórico}

El estudio de la rentabilidad siempre ha sido objeto de mucho interés no solo para los propietarios de las empresas sino también para investigadores de todo el mundo (Le et al., 2020). La rentabilidad se define como la capacidad que tiene una empresa para generar beneficios (Ali et al., 2019).

La generación constante de rentabilidad en el corto plazo es de vital importancia para que las organizaciones eviten escenarios de insolvencia financiera y posterior fracaso empresarial. Por lo general, la mayor preocupación de la dirección de una empresa es la obtención de recursos económicos que faciliten a la empresa generar beneficios. Estos recursos económicos se pueden obtener a través de fuentes internas como el aporte de accionistas o por medio de fuentes externas como préstamos. A menudo, las empresas combinan fuentes internas y externas para financiar sus operaciones y aumentar su rentabilidad (Ali et al., 2018). 
La rentabilidad se calcula mediante el uso de ratios financieros. De acuerdo con la literatura, los más utilizados por los investigadores son la rentabilidad financiera o rendimiento sobre el patrimonio y la rentabilidad económica o rendimiento sobre los activos (Adekunle, 2011; Akinlo, 2012; Miji y Jak, 2017; Susilo et al., 2020; Zygmunt, 2013). Estas razones financieras expresan la capacidad que tiene una empresa de generar ganancias a partir de los ingresos, los activos y el patrimonio de los accionistas.

Sin embargo, la mayor motivación de los investigadores es determinar los factores que inciden en la rentabilidad de las empresas. Algunos autores (Dahmash et al., 2021; Mudjijah, 2017; Parra, 2011; Prijadi y Desiana, 2017) clasifican los factores que inciden en la rentabilidad en tres categorías: (i) variables internas de la empresa: en lo que destacan los indicadores financieros, número de trabajadores; (ii) variables relacionadas al entorno de la empresa: en esta categoría resaltan la ubicación geográfica, la industria a la que pertenece la empresa e indicadores macroeconómicos, finalmente (iii) variables asociadas a la capacidad de gestión de los accionistas: en esta categoría se consideran la instrucción académica, género, estado civil, entre otras.

La mayoría de las investigaciones utilizan información financiera y contable para explicar el comportamiento de la rentabilidad (Floros y Voulgaris, 2016; Ghosh y Guha, 2015; Steinerowska-Streb, 2012; Wood, 2006). Uno de los ratios más utilizados para analizar la rentabilidad es la razón de liquidez corriente (Ching et al., 2011; Kumaraswamy, 2016; Madhou et al., 2015; Manan, 2010). Para Miji \& Jak (2017), Yameen et al. (2019) y Almaqtari et al. (2019) la solvencia a corto plazo representado por el ratio de liquidez corriente tiene una relación positiva con la rentabilidad. Al contrario de Asche et al. (2018) y Eljelly (2004) quienes afirman que la liquidez tiene una relación negativa con el rendimiento .

El endeudamiento también es considerado un indicador importante al momento de explicar la rentabilidad. Investigaciones previas (Goldmann, 2017; Sharma y Kumar, 2011) concluyen que el endeudamiento tiene una relación inversa con la rentabilidad. En cambio otros estudios concluyen que mientras mayor sea el nivel de endeudamiento, mayor es la rentabilidad (SalazarMosquera, 2017).

Otro grupo de investigadores (Afeef, 2011; Baños-Caballero et al., 2014; Kartikasary et al., 2021; Madhou et al., 2015; Mursalini et al., 2017; Nabi, 2018; Stephen y Elvis, 2011) han explorado la incidencia del capital de trabajo y sus componentes entre ellos la rotación de activos totales y el impacto de los gastos administrativos en la empresa.

\section{Indicadores financieros que inciden en la rentabilidad de las empresas}

\section{Variable Dependiente}

\section{Rentabilidad Económica}

La rentabilidad económica ha sido utilizada en la literatura como variable dependiente para analizar el desempeño empresarial (Ali et al., 2019; Chowdhury y Rasid, 2015; Madhou et al., 
2015; Sanchez, 1994). Se define como rentabilidad económica como la razón entre el beneficio neto después de impuestos dividido para el total de activos.

\section{Variables Independientes}

\section{Endeudamiento}

La razón de endeudamiento (denotado como Debt), calculada como los pasivos totales dividido para los activos totales de la compañía. Al momento de analizar el desempeño empresarial, el endeudamiento es uno de los factores que tiene mayor relevancia; en muchos casos su incidencia en la rentabilidad depende de la interacción que tenga con los factores externos de la empresa como son el tipo de sector al que pertenecen y su ubicación geográfica (Akoto et al., 2013; Islam y Ullah, 2020; Ngo et al., 2020). Los resultados de estudios anteriores con frecuencia encuentran una incidencia positiva del endeudamiento sobre la rentabilidad económica (SalazarMosquera, 2017). En cambio, otras investigaciones suelen encontrar que el nivel de deuda de las empresas tienen una relación negativa con el rendimiento económico (Holz, 2002; Lin y Rowe, 2006; Shahnia et al., 2020).

\section{Liquidez Corriente}

El nivel de solvencia en el corto plazo es sin duda, fundamental a la hora de analizar el nivel de rentabilidad de las empresas. Un manejo adecuado de la liquidez por parte de la administración de la empresa ayuda a evitar escenarios de estrés financiero y posterior fracaso empresarial. Investigaciones previas señalan que el nivel de liquidez tiene una relación inversa con la rentabilidad de las compañías (Asche et al., 2018; Eljelly, 2004; Shahnia et al., 2020). Por su parte Yameen et al. (2019), Almaqtari et al. (2019) y Goldmann (2017) coinciden que la solvencia a corto plazo tiene una relación directa y significativa sobre el nivel de rentabilidad que generan las empresas. Para este estudio se estimó el logaritmo natural del ratio de liquidez corriente con la finalidad de normalizar esta variable explicativa.

\section{Tamaño}

La literatura señala que uno de los factores más importantes al momento de analizar la rentabilidad de las empresas es su tamaño que puede estar expresado en términos del monto de sus activos, el nivel de sus ingresos o la cantidad de empleados que poseen en su nómina (Akbas y Karaduman, 2012; Akinlo, 2012; Becker-Blease et al., 2010; Budisaptorini et al., 2019; Chen y Chang, 2010). La teoría sugiere que las grandes empresas tienen más posibilidades de acceder a los mercados de capitales y de esta manera obtener mejores tasas de interés ya que aprovechan las economías de escala. En la actualidad no existe un consenso acerca de la influencia del tamaño en la rentabilidad de las empresas. Para Yazdanfar et al. (2013), Akinlo (2012) y Budisaptorini et al. (2019) el tamaño tiene una relación positiva y significativa con la rentabilidad. En cambio, para Asche et al. (2018), Goddard et al. (2005) y Akbas y Karaduman (2012) el tamaño de la empresa tiene una relación negativa y significativa con el rendimiento económico. En el presente trabajo, el tamaño de la empresa se calcula como el logaritmo natural del nivel de sus activos.

\section{Capital de Trabajo}

Esta obra se comparte bajo la licencia Creative Common Atribución-No Comercial 4.0 International (CC BY-NC 4.0) Revista de la Universidad Internacional del Ecuador. URL: https://www.uide.edu.ec/ 
Los hallazgos de estudios anteriores muestran que un adecuado manejo de las cuentas corrientes de la empresa ayudan a la generación de rentabilidad (Ching et al., 2011; Kartikasary et al., 2021; Kumaraswamy, 2016; Madhou et al., 2015; Nabi, 2018). Investigaciones previas señalan que las cuentas que pertenecen al capital de trabajo como el ciclo de conversión de efectivo y sus componentes tienen una incidencia significativamente positiva con la rentabilidad (Phuong y Hung, 2020; Quy y Nguyen, 2020; Sharma y Kumar, 2011). Por el contrario, otras investigaciones señalan que la administración de las cuentas del capital de trabajo tienen una relación negativa y significativa con el rendimiento (EL-Ansary y Al-Gazzar, 2020; Osuji y Agbada, 2020)De acuerdo con la teoría, el capital de trabajo se define como la diferencia entre los activos y los pasivos circulantes que posee una empresa y este es el criterio utilizado en el presente estudio.

\section{Rotación de activos}

La rotación de activos es considerada un indicador de eficiencia, calculado a partir de la división de las ventas para el total de activos, indica el número de veces que los activos generan ingresos en la compañía. Algunos investigadores coinciden que la rotación de activos tiene un efecto positivo en el rendimiento de las empresas (Abdulla, 2020; Akoto et al., 2013; Jamali, 2012). Por otro lado, (Latha y Rao, 2017), (Aulová et al., 2019) y (Endri et al., 2020) señalan que existe una relación negativa y significativa entre la rentabilidad y la rotación de activos. Existen también estudios que revelan que este indicador no incide en la rentabilidad de las empresas (Shahnia et al., 2020)

\section{Impacto de gastos administrativos}

El nivel de utilidad de una empresa puede ser afectado por la presencia de un elevado nivel de gastos. La necesidad de anticiparse a escenarios de insolvencia financiera hace que este indicador tenga cada vez más importancia (Abdullah, 2021; Hurley et al., 2002). Para este estudio el impacto de gastos administrativos se calcula como el monto de gastos administrativos dividido para el monto de ingresos que tuvo la compañía.

La mayoría de los estudios mencionados anteriormente se han realizado en diferentes países y han considerado diferentes tipos de empresas y sectores lo que ha provocado que sus resultados no coincidan. El presente estudio pretende explicar el comportamiento de la rentabilidad económica de las microempresas que se dedican a actividades de comercio al por mayor y menor; reparación de vehículos, automotores y motocicletas y que tienen su domicilio tributario en la provincia del Guayas.

A partir de la revisión literaria, se puede concluir que la rentabilidad se puede explicar mediante variables financieras calculados a partir de la información financiera y contable proveniente de los estados financieros. En base a lo expuesto, se plantean las siguientes hipótesis:

Hipótesis 1 (H1): El endeudamiento tiene una relación inversa con la rentabilidad económica

Hipótesis 2 (H2): El nivel de liquidez tiene una incidencia significativa en la generación de rentabilidad 
Hipótesis 3 (H3): El tamaño de las empresas, expresado en el monto de sus activos, tiene una relación negativa con el rendimiento sobre activos

Hipótesis 4 (H4): El capital de trabajo incide de manera positiva y significativa con la rentabilidad económica

Hipótesis 5 (H5): La rotación de activos influye de forma directa en el rendimiento sobre activos

\section{Metodología}

En este apartado se describe un modelo empírico de los factores que determinan la rentabilidad económica de las microempresas que pertenecen al sector $\mathrm{G}$ domiciliadas en la provincia del Guayas. Se escogieron datos de corte transversal tomando como periodo de análisis el año 2019. La especificación del modelo empírico es la siguiente:

$$
\begin{gathered}
\text { LnROA }=\beta_{0}+\beta_{1} \text { Debt }+\beta_{2} \text { LiqCte }+\beta_{3} \text { LnAct }+\beta_{4} \text { CapTrab }+\beta_{5} \text { RotacAct } \\
+\beta_{6} \text { LnGtosAdm }+\varepsilon
\end{gathered}
$$

Donde,

$\beta_{0}, \beta_{1}, \beta_{2}, \beta_{3}, \beta_{4}, \beta_{5}, \beta_{6}$ son los coeficientes de las variables que explican el modelo y $\varepsilon$ es el término de error.

\section{Selección de la muestra}

Para este estudio se utilizó información contable y financiera proveniente de los estados financieros que reportaron las empresas a la Superintendencia de Compañías, Valores y Seguros en el año 2019, institución que registra una amplia base de datos de todas las compañías en Ecuador.

La población objetivo de este trabajo fueron las microempresas que no cotizan en bolsa, registran domicilio tributario en la provincia del Guayas y que pertenecen al sector $\mathrm{G}$ que de acuerdo con la estructura esquemática de la clasificación nacional de actividades económicas (CIIU Revisión 4.0) son aquellas organizaciones que se dedican a actividades de comercio al por mayor y menor; reparación de vehículos, automotores y motocicletas.

De acuerdo con la clasificación de empresas, según su tamaño, una microempresa es aquella organización que tiene ingresos menores a USD100 mil y cuenta con una nómina entre uno a diez empleados. El procedimiento de selección y tratamiento de los datos fue el siguiente: (i) se escogieron empresas constituidas como sociedad anónima que se encontraron activas en el año 2019; (ii) se eliminaron de la muestra las empresas con datos perdidos y/o con observaciones atípicas; (iii) se consideraron a aquellas microempresas cuyo patrimonio fue mayor o igual a USD800 y (iv) que hayan generado una utilidad positiva (ganancias) durante el periodo escogido.

\section{Descripción de variables}

En el análisis empírico realizado, la variable dependiente es la rentabilidad económica que es de tipo continua y las variables independientes, asimismo de tipo continua, fueron factores 
propios de la empresa generados a partir de los estados financieras que las microempresas reportaron en el año 2019. La Tabla 1 presenta un resumen de las variables empleadas y sus definiciones.

Tabla 1

Definición de variables

\begin{tabular}{ccc}
\hline Abreviación & Variable & Definición \\
\hline ROA & Rentabilidad económica & Utilidad neta/Activos Totales \\
Debt & Endeudamiento & Pasivos Totales/Activos Totales \\
LiqCte & Liquidez Corriente & Activos Corrientes/Pasivos Corrientes \\
Act & Activos & Total de Activos \\
CapTrab & Capital de Trabajo & Activos Corrientes - Pasivos Corrientes \\
RotacAct & Rotación de Activos & Ventas/Activos Totales \\
GtosAdmVtas & Impacto de Gastos Administrativos & Gastos Administrativos/Ventas \\
\hline
\end{tabular}

Fuente: Elaboración propia

\section{Resultados y Discusión}

La Tabla 2 muestra un análisis descriptivo de las microempresas que fueron objeto de estudio. Como se puede observar, se han escogido los factores que se estiman en el modelo además de otras variables que pueden ser de interés y que ayudará a entender el comportamiento del sector escogido. Las microempresas que pertenecen al sector $\mathrm{G}$ tienen una liquidez corriente promedio de USD47 por cada dólar de deuda en el corto plazo, existen empresas que no poseen liquidez y un máximo de USD7,609.17 para cumplir con sus obligaciones circulantes. El nivel de endeudamiento es, en promedio, de 53.97\%. Existen empresas que no financian sus activos con deuda y el máximo de financiamiento es del $100 \%$. El promedio de capital de trabajo para las empresas de este sector es de USD2,560.34, el monto mínimo de este rubro es de -USD1,745,803 y un máximo de USD1,085,369 esto indica que hay empresas de este sector que tienden a recurrir a préstamos a terceros para poder operar. El promedio de la rotación de activos es de 2.13 veces, un mínimo de 0 y un máximo de 58.01 veces. El impacto de gastos administrativos tiene un promedio de $69.85 \%$ un mínimo de $0 \%$ y un máximo de $97.71 \%$. Es importante destacar que el promedio de activos que poseen las empresas que pertenecen al sector comercio es de USD74,562.76; un nivel mínimo de USD804.94 y un máximo de USD2,336,487. En cuanto a los niveles de rentabilidad, se puede apreciar que en promedio la rentabilidad económica es de $62.12 \%$ mientras que la rentabilidad financiera es de $49.97 \%$. Existen empresas que tienen un $0 \%$ de rentabilidad y un máximo de $456.81 \%$ de rentabilidad económica; el mínimo y máximo de rentabilidad financiera es de $1.14 \%$ y $382.56 \%$ respectivamente.

\section{Tabla 2}

Análisis descriptivo de la muestra

\begin{tabular}{lccccc}
\hline \multicolumn{1}{c}{ Variable } & Media & Mediana & Mínimo & Máximo & Desv. Estándar \\
\hline ROA & 0.6212 & 0.0725 & 0 & 4.5681 & 0.3022 \\
ROE & 0.4997 & 0.2602 & 0.0114 & 3.8256 & 0.6386 \\
Endeudamiento & 0.5397 & 0.6193 & 0 & 0.9992 & 0.3472
\end{tabular}


Factores explicativos de la rentabilidad de las microempresas: Un estudio aplicado al sector comercio

\begin{tabular}{lccccc}
\hline \multicolumn{1}{c}{ Variable } & Media & Mediana & Mínimo & Máximo & Desv. Estándar \\
\hline Liquidez Corriente & 47.734 & 2.0639 & 0 & $7,609.174$ & 389.7975 \\
Capital de Trabajo & $2,560.34$ & $5,288.509$ & $-1,745,803$ & $1,085,369$ & $125,070.20$ \\
Rotación de Activos & 2.1337 & 0.9807 & 0 & 58.0106 & 3.8883 \\
Impacto de Gastos & 0.6985 & 0.4064 & 0 & 0.9771 & 1.5746 \\
Activos Totales & $74,562.76$ & $28,966.84$ & 804.9399 & $2,336,487$ & $173,648.30$ \\
\hline
\end{tabular}

Fuente: Elaboración propia

Estos resultados claramente muestran la heterogeneidad que existe en las empresas del sector. Por un lado, se presume que, debido al dinamismo existente en este conjunto de compañías, el nivel de liquidez de las empresas debería ser homogénea lo que permitiría que los niveles de endeudamiento sean bajos. Sin embargo, los datos seleccionados de la muestra contradicen esta hipótesis. La capacidad de operación de operación de estas empresas, reflejada en el capital de trabajo muestra que las microempresas en la provincia de Guayas tienden a recurrir a préstamos de corto plazo para cumplir con sus obligaciones circulantes.

Un aspecto importante para destacar es que la utilidad generada por las aportaciones de los accionistas, medida por la rentabilidad financiera es alta; lo que respaldaría la hipótesis de que se constituyan nuevas empresas en este sector por lo atractivo de su rentabilidad. De hecho, el sector comercio sector inmobiliario son los grupos con mayor número de empresas en la provincia del Guayas.

La Tabla 3 muestra los resultados para determinar la relación que tienen las variables independientes usadas en el modelo con la rentabilidad económica. Los resultados indican que la rentabilidad económica está positiva y débilmente correlacionado con la rotación de activos $(r=$ 0.2998; $p<0.01)$, la liquidez corriente $(r=0.2469 ; p<0.01)$ y el impacto de los gastos administrativos y de ventas $(r=0.0820 ; p<0.05)$. Los resultados también muestran que el endeudamiento $(r=-0.4722 ; p<0.01)$ y el tamaño de la empresa definido como el total de activos $(r=-0.4312 ; p<0.01)$ están negativa y moderadamente relacionados con la rentabilidad. Finalmente se puede observar que el capital de trabajo $(r=-0.0972 ; p<0.05)$ se encuentra negativa y débilmente relacionado con el rendimiento.

\section{Tabla 3}

Análisis de correlación

\begin{tabular}{ccc}
\hline \multirow{2}{*}{ Variable } & \multicolumn{2}{c}{ Rentabilidad Económica } \\
\cline { 2 - 3 } & $\begin{array}{c}\text { Coeficiente de correlación de } \\
\text { Pearson }(\mathbf{r})\end{array}$ & P-valor \\
\hline Debt & -0.4722 & 0.0000 \\
RotacAct & 0.2998 & 0.0000 \\
LnLiqCte & 0.2469 & 0.0000 \\
LnAct & -0.4312 & 0.0000 \\
LnGtosAdmVtas & 0.0820 & 0.0283 \\
LnCapTrab & -0.0972 & 0.0128 \\
\hline
\end{tabular}

Fuente: Elaboración propia 
La Tabla 4 presenta las estadísticas resumidas de la ecuación de regresión estimada. Los resultados muestran que la ecuación de regresión es estadísticamente significativa la 0.01 $(p<0.01)$, lo que confirma que existe una relación entre el rendimiento sobre los activos y alguna o todas las variables explicativas. El valor de R-cuadrado es de 0.5127 , lo que significa que las seis variables independientes en su conjunto explican el $51.27 \%$ de la variación de la variable explicada (rendimiento sobre los activos). Por tanto, el efecto del endeudamiento, la rotación de activos, la liquidez corriente, el tamaño de la empresa medido como el monto de activos, el impacto de los gastos administrativos y de ventas y el capital de trabajo sobre el rendimiento del activo es moderado. Si se toma en cuenta los coeficientes de regresión, los resultados indican que las variables utilizadas para explicar la generación de rentabilidad son significativas al 0.05 $(p<0.05)$. El hecho que los coeficientes del endeudamiento, liquidez corriente y el nivel de activos (tamaño) sean negativos significa que un aumento de estas variables provocaría una disminución en la generación de rentabilidad. En cambio, dado que los coeficientes del capital de trabajo, rotación de activos totales y el impacto del gasto administrativo y ventas sean positivos indica que un aumento en estos factores provocaría un aumento en la rentabilidad sobre el activo.

\section{Tabla 4}

Regresión estimada

\begin{tabular}{lccc}
\hline \multicolumn{1}{c}{ Variable } & Coeficiente & Valor t & P-valor \\
\hline Debt & -1.6738 & -7.79 & 0.0000 \\
LnLiqCte & -0.2875 & -6.13 & 0.0000 \\
LnAct & -0.5293 & -6.76 & 0.0000 \\
LnCapTrab & 0.3279 & 4.87 & 0.0000 \\
RotacAct & 0.0322 & 2.14 & 0.0330 \\
LnGtosAdmVtas & 0.1013 & 2.82 & 0.0050 \\
Constante & 0.0823 & 0.16 & 0.8690 \\
F & 46.57 & & 0.0000 \\
R-cuadrado & 0.5196 & & \\
R-cuadrado ajustado & 0.5127 & & \\
\hline
\end{tabular}

Fuente: Elaboración propia

Para comprobar la ausencia de multicolinealidad entre las variables independientes se procedió a encontrar el factor de inflación de la varianza. La Tabla 5 muestra que el promedio del factor de inflación de la varianza es menor a 5 por lo que se concluye que no existe correlación entre las variables independientes.

\section{Tabla 5}

Factor de inflación de la varianza

\begin{tabular}{ll}
\hline \multicolumn{1}{c}{ Variable } & VIF \\
\hline LnAct & 4.00 \\
LnCapTrab & 3.16
\end{tabular}




\begin{tabular}{ll}
\hline \multicolumn{1}{c}{ Variable } & VIF \\
\hline Debt & 1.89 \\
LnLiqCte & 1.71 \\
RotacAct & 1.49 \\
LnGtosAdmVtas & 1.30 \\
\multicolumn{1}{c}{ Mean VIF } & 2.26 \\
\hline
\end{tabular}

Fuente: Elaboración Propia

\section{Conclusiones}

El presente estudio examina la relación que existe entre la rentabilidad económica y los factores propios (indicadores financieros) de las microempresas que pertenecen al sector $\mathrm{G}$ de la provincia del Guayas. Para esta investigación se seleccionaron 788 microempresas que reportaron sus estados financieros a la Superintendencia de Compañías, Valores y Seguros en el año 2019.

Los resultados de esta investigación muestran que el endeudamiento tiene una relación negativa y significativa con la rentabilidad económica. Este hallazgo coincide con los resultados presentados por Shahnia et al. (2020), Holz (2002) y Lin y Rowe (2006). Este estudio también muestra que la liquidez corriente y el nivel de activos inciden de forma negativa en el rendimiento sobre el activo. Este resultado coincide con Asche et al. (2018) y Eljelly (2004).

Es importante destacar que la rotación de activos totales y el capital de trabajo tiene una incidencia positiva con la rentabilidad tal como concluyen Akoto et al. (2013), Jamali (2012) y Abdulla (2020) en sus resultados.

Esta investigación no está exenta de limitaciones. En primer lugar, este trabajo solo ha considerado 788 empresas de un solo sector de la economía por lo que sus resultados no se pueden generalizar para el resto de los sectores y empresas de mayor tamaño. En segundo lugar, solo se ha considerado la información contable y financiera de las empresas que han sido objeto de estudio, se sugiere que para futuros trabajos se incluyan factores asociados al entorno de la empresa como la ubicación geográfica e indicadores macroeconómicos como el riesgo país, crecimiento del PIB y tasa de inflación.

Las conclusiones de este estudio permiten ofrecer directrices a los accionistas y dueños de empresas que permitan evaluar su gestión y cómo ésta se ve reflejada en la rentabilidad de tal manera que puedan tomar decisiones que ayuden a aumentar el rendimiento.

\section{Referencias bibliográficas}

Abdulla, Y. (2020). Firms' profitability: evidence from Bahrain and Qatar Yomna Abdulla. International Journal of Economic and Business Research, 19(1), 70-87.

Abdullah, A. A. H. (2021). Cost stickiness and firm profitability: A study in Saudi Arabian industries. Investment Management and Financial Innovations, 18(3), 327-333. https://doi.org/10.21511/imfi.18(3).2021.27

Adekunle, B. (2011). Determinants of microenterprise performance in Nigeria. International Small 
Business Journal, 29(4), 360-373. https://doi.org/10.1177/0266242610369751

Afeef, M. (2011). Analyzing the Impact of Working Capital Management on the Profitability of SME's in Pakistan. International Journal of Business and Social Science, 2(22), 173-183. http://www.ijbssnet.com/journals/Vol_2_No_22_December_2011/20.pdf

Akbas, H. E., \& Karaduman, H. A. (2012). The effect of firm size on profitability: An empirical investigation on Turkish manufacturing companies. European Journal of Economics, Finance and Administrative Sciences, 55, 21-27.

Akinlo, A. E. (2012). Firm size-profitability nexus: Evidence from panel data for Nigeria. Economic Research, 25(3), 706-721. https://doi.org/10.1080/1331677X.2012.11517530

Akoto, R. K., Awunyo-Vitor, D., \& Angmor, P. L. (2013). Working capital management and profitability: Evidence from Ghanaian listed manufacturing firms. Journal of Economics and International Finance, 5(9), 373-379. https://doi.org/10.5897/jeif2013.0539

Ali, M. M., Bakar, R. A., \& Ghani, E. K. (2018). The Effect of Firm Internal and External Characteristics on Risk Reporting Practices among Malaysian Listed Firms. Indonesian Journal of Sustainability Accounting and Management, 2(2), 107. https://doi.org/10.28992/ijsam.v2i2.53

Ali, M. M., Hussin, N. N. A. N., \& Ghani, E. K. (2019). Liquidity, growth and profitability of nonfinancial public listed Malaysia: A Malaysian evidence. International Journal of Financial Research, 10(3), 194-202. https://doi.org/10.5430/ijfr.v10n3p194

Almaqtari, F. A., Al-Homaidi, E. A., Tabash, M. I., \& Farhan, N. H. (2019). The determinants of profitability of Indian commercial banks: A panel data approach. International Journal of Finance and Economics, 24(1), 168-185. https://doi.org/10.1002/ijfe.1655

Asche, F., Sikveland, M., \& Zhang, D. (2018). Profitability in Norwegian salmon farming: The impact of firm size and price variability. Aquaculture Economics and Management, 22(3), 306-317. https://doi.org/10.1080/13657305.2018.1385659

Aulová, R., Pánková, L., \& Rumánková, L. (2019). Analysis of selected profitability ratios in the agricultural sector. Agris On-Line Papers in Economics and Informatics, 11(3), 3-12. https://doi.org/10.7160/aol.2019.110301

Baños-Caballero, S., García-Teruel, P. J., \& Martínez-Solano, P. (2014). Working capital management, corporate performance, and financial constraints. Journal of Business Research, 67(3), 332-338. https://doi.org/10.1016/j.jbusres.2013.01.016

Becker-Blease, J. R., Kaen, F. R., Etebari, A., \& Baumann, H. (2010). Employees, firm size and profitability in U.S. manufacturing industries. Investment Management and Financial Innovations, 7(2), 119-132.

Budisaptorini, A. T., Chandrarin, G., \& Asih, P. (2019). The effect of company size on company profitability and company value: The case of manufacturing companies. International Journal of Economics and Business Administration, 7(2), 249-254.

Chen, Y. S., \& Chang, K. C. (2010). Analyzing the nonlinear effects of firm size, profitability, and employee productivity on patent citations of the US pharmaceutical companies by using artificial neural network. Scientometrics, 82(1), 75-82. https://doi.org/10.1007/s11192009-0034-X

Ching, H. Y., Novazzi, A., \& Gerab, F. (2011). Relationship betwee working capital management and profitability in Brazilian listed companies. Journal of Global Business and Economics, 3(1), 74-86. https://doi.org/10.4018/978-1-5225-8970-9.ch011

Chowdhury, M. A. F., \& Rasid, M. E. S. M. (2015). The determinants of the profitability of Islamic banks: a cross-sectional study from Asia and Africa. Int. J. Business and Globalization, 
15(3), 375-388. https://doi.org/10.1504/IJBG.2015.071913

Dahmash, F., Al Salamat, W., Masadeh, W. M., \& Alshurafat, H. (2021). The effect of a firm's internal factors on its profitability: Evidence from Jordan. Investment Management and Financial Innovations, 18(2), 130-143. https://doi.org/10.21511/IMFI.18(2).2021.11

EL-Ansary, O., \& Al-Gazzar, H. (2020). Working capital and financial performance in MENA region. Journal of Humanities and Applied Social Sciences, ahead-of-p(ahead-of-print). https://doi.org/10.1108/jhass-02-2020-0036

Eljelly, A. M. A. (2004). Liquidity - profitability tradeoff: An empirical investigation in an emerging market. International Journal of Commerce and Management, 14(2), 48-61. https://doi.org/10.1108/10569210480000179

Endri, E., Lisdawati, Susanti, D., Hakim, L., \& Sugianto, S. (2020). Determinants of profitability: Evidence of the pharmaceutical industry in Indonesia. Systematic Reviews in Pharmacy, 11(6), 587-597. https://doi.org/10.31838/srp.2020.6.89

Evans, D. S. (1987). The relationship between firm growth, size and age: Estimates for 100 manufacturing industries. The Journal of Industrial Economics, 35(4), 567-581. https://doi.org/10.2307/2098578

Floros, C., \& Voulgaris, F. (2016). Efficiency, leverage and profitability: The case of Greek manufacturing sector. Global Business and Economics Review, 18(3-4), 385-401. https://doi.org/10.1504/GBER.2016.076239

Franco, M., Haase, H., \& António, D. (2020). Influence of failure factors on entrepreneurial resilience in Angolan micro, small and medium-sized enterprises. International Journal of Organizational Analysis. https://doi.org/10.1108/IJOA-07-2019-1829

Garg, V., Tewari, P., \& Srivastav, S. (2018). Liquidity and profitability analysis of selected automobile companies. International Journal of Supply Chain Management, 7(4), 101110. https://ojs.excelingtech.co.uk/index.php/IJSCM/article/view/2133

Ghosh, C., \& Guha, S. (2015). Determinants of profitability of the microenterprises led by women micro entrepreneurs: Evidence from Mumbai slums. International Journal of Entrepreneurship and Small Business, 24(4), 455-473. https://doi.org/10.1504/IJESB.2015.068631

Goddard, J., Tavakoli, M., \& Wilson, J. O. S. (2005). Determinants of profitability in European manufacturing and services: Evidence from a dynamic panel model. Applied Financial Economics, 15(18), 1269-1282. https://doi.org/10.1080/09603100500387139

Goldmann, K. (2017). Financial Liquidity and Profitability Management in Practice of Polish Business. Eurasian Studies in Business and Economics, 4, 103-112. https://doi.org/10.1007/978-3-319-39919-5_9

Handoko, B. L., Muljo, H. H., \& Lindawati, A. S. L. (2019). The effect of company size, liquidity, profitability, solvability, and audit firm size on audit delay. International Journal of Recent Technology and 8ngineering, 8(3), 6252-6258. https://doi.org/10.35940/ijrte.C5837.098319

Holz, C. A. (2002). The impact of the liability - Asset ratio on profitability in China's industrial state-owned enterprises. China Economic Review, 13(1), 1-26. https://doi.org/10.1016/S1043-951X(01)00054-2

Hurley, M. W., Touran, A., \& Asce, M. (2002). Cost Structure and Profitability of Design Services Industry. October, 167-172.

Islam, M., \& Ullah, G. M. W. (2020). Debt and profitability: Evidence from Bangladesh. International Journal of Monetary Economics and Finance, 13(4), 362-382. 
https://doi.org/10.1504/ijmef.2020.109995

Jamali, A. H. (2012). Management efficiency and profitability in Indian automobile industry: from theory to practice. Indian Journal of Science and Technology, 5(5), 2779-2781.

Jamil, S. A., Al Ani, M. K., \& Al Shubiri, F. N. (2015). The effect of working capital management efficiency on the operating performance of the industrial companies in Oman. International Journal of Economics and Financial Issues, 5(4), 897-904.

Janda, K., Rausser, G., \& Strielkowski, W. (2011). Determinants of profitability of Polish rural micro-enterprises. Economic Policy, 2116, 0-33.

Kartikasary, M., Marsintauli, F., Sitinjak, M., Laurens, S., Novianti, E., \& Situmorang, R. (2021). The effect of working capital management, fixed financial asset ratio, financial debt ratio on profitability in Indonesian consumer goods sector. Accounting, 7(3), 661-666. https://doi.org/10.5267/j.ac.2020.12.011

Kumaraswamy, S. (2016). Impact of working capital on financial performance of gulf cooperation council firms. International Journal of Economics and Financial Issues, 6(3), 1136-1142.

Latha, M., \& Rao, S. N. (2017). Determinants of profitability: Evidence from listed companies in the bse-fmcg. International Journal of Economic Perspectives, 11(3), 1264-1272.

Le, T. N., Mai, V. A., \& Nguyen, V. C. (2020). Determinants of profitability: Evidence from construction companies listed on Vietnam Securities Market. Management Science Letters, 10(3), 523-530. https://doi.org/10.5267/j.msl.2019.9.028

Lin, S., \& Rowe, W. (2006). Determinants of the profitability of China's regional SOEs. China Economic Review, 17(2), 120-141. https://doi.org/10.1016/j.chieco.2005.05.001

Madhou, A., Moosa, I., \& Ramiah, V. (2015). Working Capital as a Determinant of Corporate Profitability. Review of Pacific Basin Financial Markets and Policies, 18(4), 1-17. https://doi.org/10.1142/S0219091515500241

Manan, S. K. A. (2010). Empirical analysis on the relationship between debt level and SMEs profitability. 2010 International Conference on Science and Social Research, 1329-1333. https://doi.org/10.1109/CSSR.2010.5773745

Miji, K., \& Jak, D. (2017). The determinants of agricultural industry profitability: evidence from southeast Europe. 154-173.

Mudjijah, S. (2017). Working Capital Management and Profitability of Companies: Empirical Study on Corporate Sub Sectors of the Food and Drinks Listed on the Indonesia Stock Exchange. 14(4), 451-456.

Mursalini, W. I., Husni, T., \& Hamidi, M. (2017). Analysis of funding, working capital turnover, liquidity and sales growth to profitability. Advanced Science Letters, 23(9), 8341-8346. https://doi.org/10.1166/asl.2017.9889

Nabi, A. A. (2018). Impact of working capital management on financial performance of the firm. Journal of Economic Info, 5(4), 1-6. https://doi.org/10.31580/jei.v5i4.91

Ngo, V. T., Tram, T. X. H., \& Vu, B. T. (2020). The Impact of Debt on Corporate Profitability: Evidence from Vietnam. Journal of Asian Finance, Economics and Business, 7(11), 835842. https://doi.org/10.13106/jafeb.2020.vol7.no11.835

Osuji, C. C., \& Agbada, A. O. (2020). Imperatives of effective working capital management and profitability in the banking industry in Nigeria. International Journal of Financial Research, 11(2), 229-242. https://doi.org/10.5430/ijfr.v11n2p229

Parra, J. F. (2011). Determinantes de la probabilidad de cierre de nuevas firmas en Bogotá. Revista Facultad de Ciencias Económicas, XIX(1), 27-53.

Phuong, N. T. T., \& Hung, D. N. (2020). Impact of working capital management on firm 
profitability: Empirical study in Vietnam. Accounting, 6, 259-266. https://doi.org/10.5267/j.ac.2020.3.001

Prijadi, R., \& Desiana, P. M. (2017). Factors affecting the profitability and growth of Small \& Medium Enterprises (SMEs) in Indonesia. International Journal of Economics and Management, 11(SpecialIssue1), 35-44.

Quy, V. T., \& Nguyen, L. T. M. (2020). Effects of working capital management on firm performance and firm value - A study of the fisheries industry in Vietnam. Hcmcoujs $\begin{array}{llll}\text { Economics } \quad \text { and } \quad \text { Business } & \text { 4(2), }\end{array}$ https://doi.org/10.46223/hcmcoujs.econ.en.7.2.184.2017

Rasiah, D., Tong, D. Y. K., \& Kim, P. K. (2014). Profitability and firm size-growth relationship in construction companies in Malaysia from 2003 to 2010. Review of Pacific Basin Financial Markets and Policies, 17(3), 1-19. https://doi.org/10.1142/S0219091514500143

Salazar-Mosquera, G. M. (2017). Factores determinantes del desempeño financiero en el sector manufacturero en la República del Ecuador. Panorama Económico, 25(2), 243-254. https://doi.org/10.32997/2463-0470-vol.25-num.2-2017-2076

Sanchez, A. (1994). La Rentabilidad Económica Y Financiera de la Gran Empresa Española. Revista Española de Financiación y Contabilidad, XXIV N 78, 159179.

Shahnia, C., Purnamasari, E. D., Hakim, L., \& Endri, E. (2020). Determinant of profitability: Evidence from trading, service and investment companies in Indonesia. Accounting, 6(5), 787-794. https://doi.org/10.5267/j.ac.2020.6.004

Sharma, A. K., \& Kumar, S. (2011). Effect of working capital management on firm profitability: Empirical evidence from India. Global Business Review, 12(1), 159-173. https://doi.org/10.1177/097215091001200110

Steinerowska-Streb, I. (2012). The determinants of enterprise profitability during reduced economic activity. Journal of Business Economics and Management, 13(4), 745-762. https://doi.org/10.3846/16111699.2011.645864

Stephen, M., \& Elvis, K. (2011). Influence of working capital management on firms profitability: A case of smes in Kenya. In International Business Management (Vol. 5, Issue 5, pp. 279286). https://doi.org/10.3923/ibm.2011.279.286

Susilo, D., Wahyudi, S., \& Pangestuti, I. R. D. (2020). Profitability determinants of manufacturing firms in Indonesia. International Journal of Economics and Business Administration, 8(2), 53-64. https://doi.org/10.35808/ijeba/443

Vivel-Búa, M., Lado-Sestayo, R., \& Otero-González, L. (2016). Impact of location on the probability of default in the Spanish lodging industry: A study of MSMEs. Tourism Economics, 22(3), 593-607. https://doi.org/10.5367/te.2015.0461

Wood, E. H. (2006). The internal predictors of business performance in small firms. A logistic regression analysis. Journal of Small Business and Enterprises Develpment, 13(3), 441453. https://doi.org/10.1108/14626000610680299

Yameen, M., Farhan, N. H. S., \& Tabash, M. I. (2019). The impact of liquidity on firms' performance: Empirical investigation from Indian pharmaceutical companies. Academic Journal of Interdisciplinary Studies, 8(3), 212-220. https://doi.org/10.36941/ajis-20190019

Yazdanfar, D., Salman, K., \& Arnesson, L. (2013). Life cycle of profitability among Swedish micro firms. World Review of Entrepreneurship, Management and Sustainable Development, 9(3), 340-351. https://doi.org/10.1504/WREMSD.2013.054738

Zambrano Farías, F. J., Sánchez Pacheco, M. E., \& Valls Martínez, M. del C. (2021). Factors 
Explaining the Business Survival of MSMEs in Ecuador Factores Explicativos de la Supervivencia Empresarial de la Mipyme en Ecuador. Studies of Applied Economics, 39(8), 1-18. https://doi.org/10.25115/eea.v39i8.4061

Zygmunt, J. (2013). Does liquidity impact on profitability? A case of polish listed IT companies. International Journal of Advanced Research in Management and Social Sciences, 1(3), 247-251. https://bit.ly/3nPQTE9 